\title{
A CHARACTERIZATION OF HARMONIC IMMERSIONS OF SURFACES
}

\author{
BY TILLA KLOTZ MILNOR
}

Communicated by J. A. Wolf, January 3, 1977

Let $S$ be an oriented surface with Riemannian metric $d s^{2}$, and $M^{n}$ a Riemannian manifold of dimension $n \geqslant 2$. We present here a characterization of harmonic immersions $f: S \rightarrow M^{n}$ which sheds some light on their differential geometric properties. While $C^{\infty}$ smoothness is assumed throughout, less is needed.

To work on the Riemann surface determined by $d s^{2}$ on $S$, use conformal parameters $z=x_{1}+i x_{2}$ which correspond to $d s^{2}$-isothermal coordinates $x_{1}, x_{2}$ on $S$. Given any local coordinates on $M^{n}$, write $f=\left(f^{\alpha}\right)$ and $f_{i}^{\alpha}=\partial f^{\alpha} / \partial x_{i}$ where $i=1,2$ and $\alpha, \beta, \gamma=1,2, \ldots, n$. An immersion $f: S \rightarrow M^{n}$ is harmonic if and only if for each $\alpha$ and for any $d s^{2}$-isothermal coordinates $x_{1}, x_{2}$ on $S$

$$
\partial^{2} f^{\alpha} / \partial x_{i}^{2}+\Gamma_{\beta \gamma}^{\alpha} f_{i}^{\beta} f_{i}^{\gamma}=0
$$

where $\Gamma_{\beta \gamma}^{\alpha}$ are the Christoffel symbols for the metric on $M^{n}$, and one sums on the indices $\beta, \gamma$ and $i$.

To any real quadratic form $X=l_{i j} d x_{i} d x_{j}$ on $S$, associate on $R$ the quadratic differential $\Omega(X, R)$ and the conformal metric $\Gamma(X, R)$ given by $4 \Omega(X, R)$ $=\left(l_{11}-l_{22}-2 i l_{12}\right) d z^{2}$ and $2 \Gamma(X, R)=\left(l_{11}+l_{22}\right) d z d \bar{z}$ respectively. Thus $X=2 \operatorname{Re} \Omega+\Gamma$ on $R$. (See [10].) Call $\Omega(X, R)$ holomorphic if and only if the coefficient of $d z^{2}$ is complex analytic in $z$ for every conformal parameter $z$ on $R$. An immersion $f: S \rightarrow M^{n}$ yields many quadratic forms of interest, among them the induced metric I, and the second fundamental forms $\operatorname{II}(N)$ de. termined by choices of a unit normal vector field $N$.

Definition. An immersion $f: S \rightarrow M^{n}$ is $R$-minimal if and only if $\Omega(\mathrm{I}, R)$ is holomorphic, and $\Gamma(\mathrm{II}(N), R) \equiv 0$ for any choice (local or global) of a unit normal vector field $N$.

An $R$-minimal immersion is minimal if and only if $R$ is the Riemann surface $R_{\mathrm{I}}$ determined on $S$ by $\mathrm{I}$. It is known that a conformal immersion $f: S \rightarrow$ $M^{n}$ is harmonic if and only if it is minimal. Indeed, this is established in [2] independent of the dimensions of $S$ and $M^{n}$. By analogy, we have the following

AMS (MOS) subject classifications (1970). Primary 53B25, 53C40. 
THEOREM. An immersion $f: S \rightarrow M^{n}$ is harmonic if and only if it is $R$ minimal.

This result is known for maps $f: S \rightarrow M^{2}$. (See [4] for references.) It is also known that $\Omega(\mathrm{I}, R)$ must be holomorphic for any harmonic map $f: S \rightarrow M^{n}$, so that the only harmonic maps of the 2-sphere must be minimal ([2] and [8]). We consider immersions here to provide (when $n \geqslant 3$ ) a well-defined $(n-2)$-dimensional normal space everywhere.

Note that $\Gamma(\mathrm{II}(N), R) \equiv 0$ for all $N$ means that the trace of $\operatorname{II}(N)$ with respect to $d s^{2}$ vanishes for all $N$. When $d s^{2} \propto \mathrm{I}$, this condition alone forces a minimal immersion, for it says that the mean curvature vector [11, p. 13] vanishes. Indeed, by our Theorem, the "mean curvature vector" formed with $d s^{2}$ in place of I vanishes for any harmonic immersion $f: S \rightarrow M^{n}$. The converse can fail when $R \neq R_{\mathrm{I}}$. For example, if $S$ is immersed in $E^{3}$ with Gauss curvature $K \equiv$ -1 , the usual asymptotic Tchebychev coordinates [9, p. 528] are II'-isothermal, where $\sqrt{H^{2}+1} \mathrm{II}^{\prime}=H \mathrm{II}+\mathrm{I}$, with $H$ mean curvature. Here $\Gamma\left(\mathrm{II}, R_{\mathrm{II}}\right) \equiv 0$ but $\Omega\left(\mathrm{I}, R_{\mathrm{II}^{\prime}}\right)$ is not holomorphic. Similarly, $\Omega(\mathrm{I}, R)$ holomorphic does not imply $\Gamma(\mathrm{II}(N), R) \equiv 0$ for any $N$. This is obvious when $R=R_{\mathrm{I}}$. Less trivially, if $S$ is immersed in $E^{3}$ with $K \equiv 1$, then $\Omega\left(\mathrm{I}, R_{\mathrm{II}}\right) \neq 0$ is holomorphic, but $\Gamma\left(\mathrm{II}, R_{\mathrm{II}}\right) \equiv$ II does not vanish [5].

The proof of the theorem is elementary, using the Gauss equations [5, p. 160]. Some results which follow from the theorem are stated below for the special case $n=3$. Full details and proofs will appear elsewhere. Hereafter, $f: S \rightarrow M^{3}$ is an immersion with fundamental forms I and II, mean curvature $H$, Gauss curvature $K$ and intrinsic curvature $K(\mathrm{I})$. Denote by $K$ the sectional curvature of $M^{3}$ for planes tangent to $S$, by $\Lambda=g \mathrm{I}+h \mathrm{II}$ any positive definite linear combination with real valued coefficients $g$ and $h$, by $R$ the Riemann surface determined on $S$ by $d s^{2}$ and by $R$ an arbitrary Riemann surface on $S$. The form

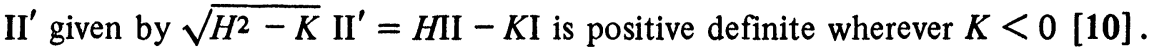
Lemmas 1 and 2 reflect the separate effects of the conditions $\Omega(I, R)$ holomorphic and $\Gamma(\mathrm{II}, R) \equiv 0$. Theorem 2 includes a correction of the Corollary to Theorem 2 in [7].

LEMmA 1. If $\Omega=\Omega(\mathrm{I}, R)$ ₹ 0 is holomorphic, then except at isolated points where $\Omega=0$, there exists a cannonically determined function $F>0$ on $S$ which is R-superharmonic where $K(\mathrm{I}) \geqslant 0$ and $R$-subharmonic where $K(\mathrm{I}) \leqslant$ $0[1, \mathrm{p} .135]$.

LEMMA 2. If $\Gamma(\mathrm{II}, R) \equiv 0$ for any one $R$ on $S$, then $K \leqslant 0$ (so that $K(\mathrm{I})$ $\leqslant K)$, and $H=0$ wherever $K=0$.

THEOREM 1. If $f: S \rightarrow M^{3}$ is harmonic with $d s^{2}=\Lambda$, then either $\Lambda \propto \mathrm{I}$, or else (except at isolated points where $\Lambda \propto \mathrm{I}) \Lambda \propto \mathrm{II}^{\prime}$. 
THEOREM 2. If $f: S \rightarrow M^{3}$ is harmonic with $d s^{2}=\mathrm{II}^{\prime}, H$ never zero and 0 丰 $K(\mathrm{I}) \leqslant 0$, then $H^{\prime} / H$ is not bounded.

THEOREM 3. If $f: S \rightarrow M^{3}$ is harmonic with $d s^{2}=\mathrm{II}^{\prime}$ complete, $|K / H|$ bounded and $K\left(\mathrm{II}^{\prime}\right) \leqslant 0$ then $K\left(\mathrm{II}^{\prime}\right) \equiv 0$.

THEOREM 4. If $f: S \rightarrow M^{3}$ is harmonic with $R$ parabolic $[1, \mathrm{p} .209]$, I nowhere proportional to $d s^{2}$ and $K(\mathrm{I}) \geqslant 0$, then $K(\mathrm{I}) \equiv 0$.

\section{REFERENCES}

1. L. V. Ahlfors and L. Sario, Riemann surfaces, Princeton Univ. Press, Princeton, N. J., 1960. MR $22 \# 5729$.

2. S. S. Chern and S. I. Goldberg, On the volume decreasing property of a class of real harmonic mappings, Amer. J. Math. 97 (1975), 133-147. MR 51 \#4102.

3. J. Eells, Jr. and J. H. Sampson, Harmonic mappings of Riemannian manifolds, Amer. J. Math. 86 (1964), 109-160. MR 29 \#1603.

4. J. Eells and J. C. Wood, Restrictions on harmonic maps of surfaces, Topology 15 (1976), 263-266.

5. L. P. Eisenhart, Riemannian geometry, Princeton Univ. Press, Princeton, N. J., 1926.

6. T. Klotz, Some uses of the second conformal structure on strictly convex surfaces, Proc. Amer. Math. Soc. 14 (1963), 793-799. MR 27 \#2917.

7. - Surfaces harmonically immersed in $E^{3}$, Pacific J. Math. 21 (1967), 79 87. MR 38 \#646.

8. L. Lemaire, Applications harmoniques de surfaces Riemanniennes, Math. Inst. Univ. Warwick, 1975 (preprint).

9. T. K. Milnor, Efimov's theorem about complete immersed surfaces of negative cur. vature, Advances in Math. 8 (1972), 474-543. MR 46 \#835.

10. Restrictions on the curvatures of $\Phi$-bounded surfaces, J. Differential Geometry 11 (1976), 31-46.

11. R. Osserman, A survey of minimal surfaces, Van Nostrand Reinhold, New York, 1969. MR 41 \#34.

DEPARTMENT OF MATHEMATICS, RUTGERS UNIVERSITY, NEW BRUNSWICK, NEW JERSEY 08903 